\title{
BINARIES IN ACCELERATION AND STOCHASTIC HIPPARCOS SOLUTIONS
}

\author{
F. ARENOU \\ DASGAL/URA 335 du C.N.R.S. - Observatoire de Paris-Meudon, \\ F-92195 Meudon Cedex - E-mail: Frederic.Arenou@obspm.fr
}

The Hipparcos astrometric parameters were obtained through the merging of abscissae data obtained by the FAST and NDAC Data Reduction Consortia. Using a different reduction scheme, other astrometric solutions may be obtained, although not statistically different: a weighted solution using FAST and NDAC astrometric solutions is shown, which gives both astrometric data and associated covariance matrix. This is applied to the acceleration and stochastic solutions. The intersection between merged and weighted solutions is then studied from the point of view of the possible signature of astrometric binaries.

New astrometric binaries were found during the Hipparcos data reduction process and their orbits were determined or improved. Other astrometric binaries may however be detected by searching for a quasi-sinusoidal signature in right ascension and declination. For this purpose the observations of the candidate stars have been combined at a few different epochs, using the Hipparcos astrometric intermediate data.

A simultaneous fit of the astrometric and orbital parameters has then been done. However in most of the cases other ground-based data is needed in order to confirm the suspected binarity and to give a reliable orbit.

\section{APERTURE SYNTHESIS OF HIPPARCOS TRANSIT DATA}

\author{
C.F. QUIST, L. LINDEGREN, S. SÖDERHJELM \\ Lund Observatory \\ Box 43, SE-221 00 Lund, Sweden
}

The ESA Hipparcos astrometry satellite did not contain an imaging detector (such as a CCD) but used a modulating grid of alternating transparent and opaque bands to modulate the light from a given object. The Fourier coefficients of the modulated detector signal were the basis for the astrometric and photometric processing leading to the Hipparcos Catalogue. These coefficients are included (for a subset of the stars) in the CD-ROM version of the Hipparcos Catalogue as the 'Hipparcos Transit Data'. In this work, the Transit Data are used in a completely different and novel application: the modulation components from a single scan are considered to be equivalent to the interference fringes from an interferometer oriented along the scan direction and having baselines of $0, \simeq 10$ and $\simeq 20 \mathrm{~cm}$ and a wavelength of $550 \mathrm{~nm}$. Using all the scans collected over the mission lifetime, it is possible to use the complex visibilities (containing both amplitude and phase information) calculated from the Transit Data to create aperture synthesis images of various type of objects such as visual or orbiting binary systems. More information, including sample images, can be found in: Quist C.F., Lindegren L., Söderhjelm S., 1997, Using Hipparcos Transit Data for Aperture Synthesis Imaging, Hipparcos Venice '97 Symposium, ESA SP-402 (in press). 